\title{
A Rocket Model of Neutrino Jet for Pulsar Kicks
}

\author{
Q. H. Peng, L. D. Zhang \& X. L. Luo \\ Department of Astronomy, Nanjing University, Nanjing 210093, China \\ C. K. Chou \\ Institute of Astronomy and Department of Physics, National Central \\ University, Chung-Li Taiwan 32054, China Taipei
}

\begin{abstract}
On the basis of the neutrino emission from the isotropic ${ }^{1} \mathrm{~S}_{0}$ neutron superfluid vortices in neutron star interiors, we propose a rocket model of neutrino jet for the observed pulsar kick.
\end{abstract}

\section{Introduction}

Ever since the discovery of pulsars in 1967 , astronomers have been very surprised to discover that the space velocities of pulsars are generally rather high. Except for the 94 known recycled pulsars the percentage of of single pulsars with velocities exceeding $100,200,300,500$ and $1000 \mathrm{~km} \mathrm{~s}^{-1}$ are, respectively, $79 \%$, $67 \%, 40 \%, 16 \%$ and $5.6 \%$. This means that high-velocity pulsars are rather common phenomena in interstellar space and the cosmos. The space velocities of these pulsars are far above both their progenitor stars and normal stars (about $20-40 \mathrm{~km} \mathrm{~s}^{-1}$ ).

At the present stage, most theories conceived by astronomers to explain the unanticipated high velocities of nascent pulsars have been established on the basis of certain spatially asymmetric causes due to fundamental physics or dynamical effects during the very short interval of the supernova explosion in which a pulsar or neutron star is formed. Until now, four different kinds of mechanisms for the observed neutron star kicks have been proposed (Arras \& Lai 1999; Kusenko \& Segre 1999; Kusenko 1999; Lai \& Qian 1998a, 1998b; Lai 2001; Lai, Chernoff \& Cordes 2001).

It seems that none of the four different kinds of mechanisms can convincingly and successfully explain the huge pulsar kicks observed. We therefore here present the idea of a gradual acceleration due to a neutrino jet rocket model for pulsar kicks.

\section{A Rocket Model of Neutrino Jet for Pulsar Kicks}

In 1982, Peng, Huang \& Huang (1982) proposed that neutrino cyclotron emission from the superfluid vortex neutrons may be used as a possible mechanism for pulsar spin-down. Starting from this theory and making use of the spatial asymmetry of neutrino spin due to parity non-conservation in weak interaction, 


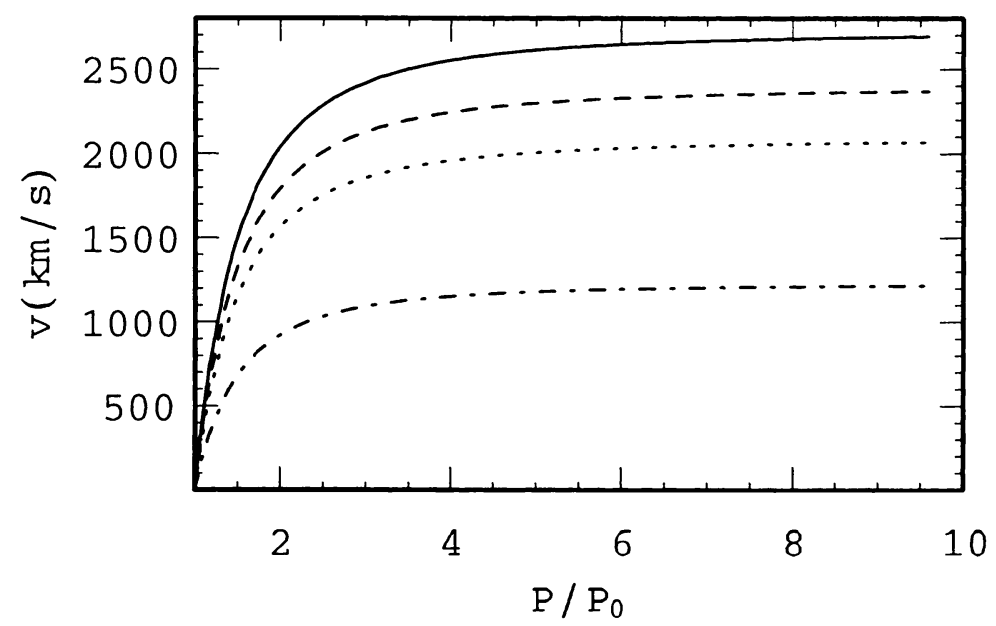

Figure 1. The acceleration curves for the neutrino jet rocket model without magnetic field decay. The spatial kick velocity of pulsars is plotted as a function of the dimensionless variable $P / P_{0}$ where $P$ and $P_{0}$ respectively denote the period and the initial period. The curves (from top to bottom) corresponds respectively to $P_{0}=0.4,0.45,0.5$ and $0.7 \mathrm{~ms}$. The parameter $\xi$ has value $1.0 \times 10^{17}$ here.

we now propose a new mechanism for pulsar kicks based on a neutrino rocket model from the superfluid vortex neutrons. The main idea is as follows.

We note that neutrons in circular motion can emit a neutrino-antineutrino pair via the neutral current in the unified theory of electro-weak interactions. Similarly, the superfluid vortex neutrons can also emit neutrinos and antineutrinos (Peng et al. 1982). The neutrons in both of these processes will lose their energy, $E_{n}^{(r o t)}$, and angular momentum, $\vec{J}_{n}$.

It is expected that the angular distributions of the neutrinos and antineutrinos are not asymmetric due to the angular momentum loss from the neutrons, although we have $N(\nu)=N(\bar{\nu})$ according to lepton conservation. More specifically, if the angular momentum of the neutron is carried away primarily by the neutrinos, the direction of the emitted neutrinos with left hand helicity must be opposite to the original neutron angular momentum, while the neutron itself will receive a recoil along the direction of its angular momentum. Fortunately, it has shown by very recent observational evidence that the kicks of two young pulsars (the Crab and Vela pulsars) are basically consistent with their spinning axes (Lai et al. 2001). Hence, it is anticipated that the angular momentum of the superfluid vortex neutrons is mainly carried away by the neutrinos rather than by antineutrinos.

\section{Pulsar Kick Generated by Neutrino Rocket Propulsion}

The neutrino luminosity due to superfluid vortex neutrons in the neutron star interior has been derived by Peng et al. (1982). Making use of their result, we 


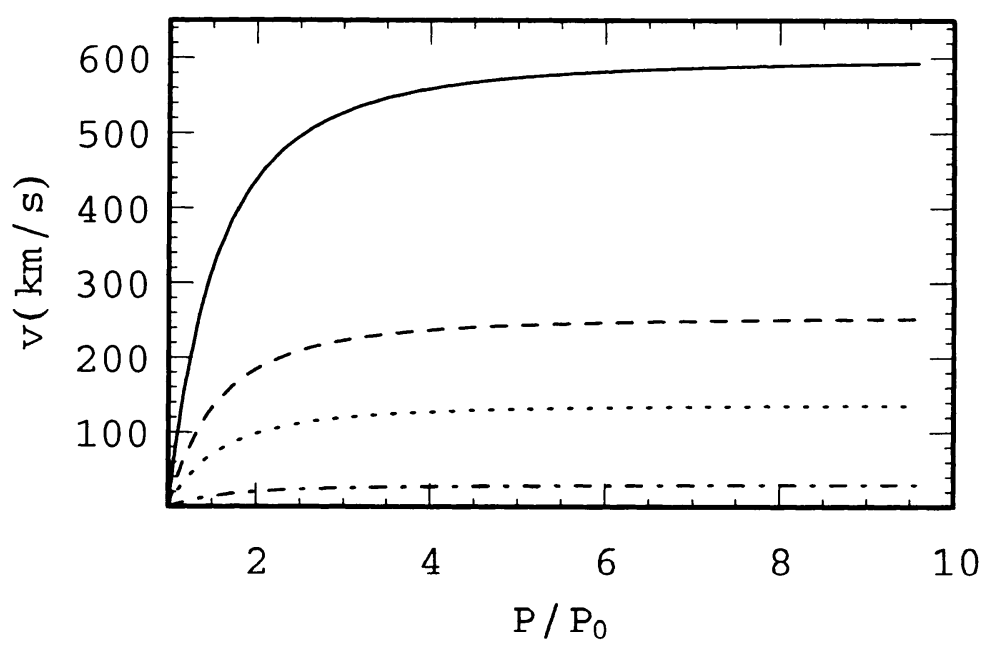

Figure 2. Ccomparison of the calculated pulsar velocity with observations in the absence of magnetic field decay. The pulsar period $P$ is treated as the independent variable here. The curves computed from our model (from top to bottom) correspond respectively to $P_{0}=1,1.5$, 2 and $4 \mathrm{~ms}$. Here the magnetic field is $10^{12}$ gauss.

may study the acceleration of the nascent neutron star and determine its kick velocity, $\vec{v}$, in terms of the rocket propulsion model. The recoil acceleration of the neutron star due to the effect of neutrino rocket propulsion as briefly discussed above may be written as $M \frac{d \vec{v}}{d t}=\eta_{\nu} \frac{W_{\nu}}{c}$, where $W_{\nu}$ is the power for neutrino emission from the superfluid vortex neutrons in the neutron star, and $M$ is the mass of the neutron star. The effective asymmetry coefficient $\eta_{\nu}$ for the neutrino emission may be estimated to be $\eta_{\nu} \approx 5 \%-20 \%$. It is found (Peng et al. 1982) that $W_{\nu}=b G(n) \Omega$, where $\Omega$ represents the rotating angular velocity of the neutron star, $b \cong 4.61 \times 10^{22} R_{6}^{3}$ (in cgs units), $R_{6}$ is the radius of the neutron star in unit of $10^{6} \mathrm{~km}$, and $G(n)=\frac{\overline{n^{7}}}{\bar{n}}$, where $n$ denotes the quantum number of the neutron superfluid vortices and decreases when the pulsar period increases during the dynamical evolution of pulsars spin down. Making a working assumption $G(n)=G\left(n_{0}\right)\left(P / P_{0}\right)^{-\beta}(\beta \approx 3)$, the corresponding recoil velocity of the neutron star may then be obtained by using the spin down law for pulsars in our hybrid model. The results for neutron star kicks with different initial periods are shown in Figures 1 and 2. In these Figures, the pulsar velocity $v$ is shown as a function of $x=P / P_{0}$ (more precisely $\left.v\left(P / P_{0}\right)-v_{0}\right)$.

We will now elaborate on some of the important and subtle points of our model. We note that the initial neutron superfluid vortex quantum number, $n_{0}$, and the initial spin period, $P_{0}$, are the two most important parameters of our model. In order for the neutron star to receive larger pulsar kicks of several hundred $\mathrm{km} \mathrm{s}^{-1}$ or even exceed $100 \mathrm{~km} \mathrm{~s}^{-1}$ from the acceleration scenario predicated by our neutrino jet rocket mechanism, it is required that the initial vortex quantum number $n_{0}$ reach 500-700 for the model without magnetic field decay, or $n_{0}=(5-8) \times 10^{3}$ for models with magnetic field decay. Such huge initial 
vortex quantum numbers are possible vis a vis the chaotic and violent process during which neutron stars are born. This is because the spinning angular velocity, $\Omega$, of the neutron star becomes much higher after the collapse due to conservation of the angular momentum of the entire star. We easily derive the result $\Omega_{0} \geq 1.0 \times 10^{4} \mathrm{~s}^{-1}, P_{0}<1 \mathrm{~ms}$. However, for a rapidly rotating stable neutron star, a considerable amount of the spinning angular momentum must have been converted into the highly chaotic and turbulent (classical) whirlpool vortices. Moreover, it is also expected that the turbulent vortices can be further converted into quantized superfluid vortices with very high initial vortex quantum number $n_{0}>10^{4}$ or more, provided that the temperature of the neutron star decreased down to $T<T_{\text {trans }}=2 \times 10^{10} \mathrm{~K}$.

\section{Main Results in Our Model}

It has been shown that our theory predicts naturally the gradual acceleration of nascent pulsars during the early stage from $P_{0}$ to $10 P_{0}$ (about 200-300 years), and that huge natal kicks exceeding $1000 \mathrm{~km} \mathrm{~s}^{-1}$ follow very nicely from our model. We have investigated the acceleration scenario during the early stage of pulsar evolution in terms of the initial period and the initial magnetic fluid. In particular: (a) the observed alignment of pulsar kicks with their spinning axes may be interpreted naturally; (b) all high velocity pulsars with spatial velocities higher than $100 \mathrm{~km} \mathrm{~s}^{-1}$ have initial periods shorter than $2-3 \mathrm{~ms}$, moreover the initial periods of those pulsars with huge kicks $\left(>1000 \mathrm{~km} \mathrm{~s}^{-1}\right)$ are shorter than $0.8 \mathrm{~ms}$; (c) for the same magnetic fields, the initial periods of high velocity pulsars are short; (d) for the same initial period, high velocity pulsars have weaker magnetic fields.

Acknowledgments. This research is supported by the National Natural Science Foundation of China (No. 10173005, 10273006 and 19935030) and a grant from the National Education Ministry of China for $\mathrm{PhD}$ candidate training (2000028417).

\section{References}

Arras, P., \& Lai, D. 1999, Phys. Rev. D, 60, 043001

Kusenko, A., \& Segre, G. 1999, Phys. Rev. D, 59, 061302

Kusenko, A. 1999, preprint (astro-ph/9903167)

Lai, D., \& Qian, Y.-Z. 1998a, ApJ, 495, L103

Lai, D., \& Qian, Y.-Z. 1998b, ApJ, 505, 844

Lai, D. 2001, in Physics of Neutron Star Interiors, eds. D. Blaschke, N. K. Glendenning, \& A. Sedrakian, (Heidelberg: Springer-Verlag), p. 424

Lai, D., Chernoff, D. F., \& Cordes, J. M. 2001, ApJ, 549, 1111

Peng, Q.-H., Huang K.-L., \& Huang, J.-H. 1982, A\&A, 107, 258 